\title{
929.
}

\section{NOTE ON THE SKEW SURFACES APPLICABLE UPON A GIVEN SKEW SURFACE.}

[From the Proceedings of the London Mathematical Society, vol. xxIII. (1892), pp. 217-225.]

THE question was considered by Bonnet, in $\S 7$ of his "Mémoire sur la théorie générale des surfaces," Jour. École Polyt., Cah. 32 (1848); I resume it here, making a greater use of the line of striction.

We may construct a skew surface, inextensible but flexible about its generating lines, as follows: Imagine a flexible extensible plane, and in it the rigid parallel lines $L, L_{1}, L_{2}, L_{3}$, \&c., connected each with the following one by the rigid lines

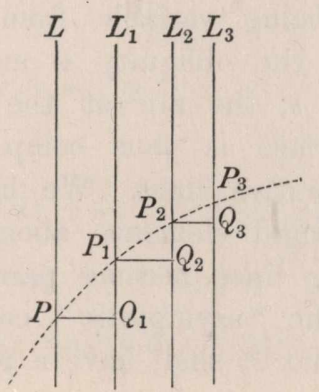

$P Q_{1}, P_{1} Q_{2}, P_{2} Q_{3}$, \&c., where $P Q_{1}$ cuts $L, L_{1}, P_{1} Q_{2}$ cuts $L_{1}, L_{2}$, \&c., at right angles ; the angles $L P P_{1}, L_{1} P_{1} P_{2}, L_{2} P_{2} P_{3}$, \&c., are taken to be $\omega, \omega_{1}, \omega_{2}$, \&c., respectively. Keeping the line $L$ fixed, we may twist the whole plane $L_{1} L_{2} L_{3} \ldots$ round $P Q_{1}$, so that the line $L_{1}$ becomes inclined at a small angle to $L$, these lines now having $P Q_{1}$ for their shortest distance, and the lines $L_{2}, L_{3}$, \&c., remaining parallel to $L_{1}$ in its new position; the foregoing twisting implies an extension (increasing with the 
distance on each side from $P$ ) of the strip or element between the lines $L, L_{1}$; after this twisting, we imagine the strip in question to become rigid. The amount of the twist is such that, if $d \phi$ be the inclination of the lines $L, L_{1}$, we have $d \phi=P Q_{1} \div \dot{\tau}$, viz. the twist $d \phi$ is in a certain proportion to the shortest distance $P Q_{1}$. Similarly, keeping the line $L_{1}$ fixed, we twist the whole plane $L_{2} L_{3} \ldots$ round $P_{1} Q_{2}$, the amount of the twist or inclination $d \phi$ of the lines $L_{1}$ and $L_{2}$ being $=P_{1} Q_{2} \div \tau_{1}$; after the twist, we imagine the strip or element between the two lines $L_{1}, L_{2}$ to become rigid. Proceeding in this manner, we have the series of rigid elements $L L_{1}, L_{1} L_{2}, L_{2} L_{3}$, \&c.; putting the line $L$ in any given position, and then keeping it fixed, we may turn the element $L L_{1}$ round this line so as to bring $L_{1}$ into a certain position; then, keeping $L_{1}$. fixed in this position, we may turn the element $L_{1} L_{2}$ round $L_{1}$ so as to bring $L_{2}$ into a certain position, and so on. To explain this further, imagine through a point $O$ a series of lines $K, K_{1}, K_{2}, K_{3}$, \&c., such that the inclination of $K$ and $K_{1}$ is equal to that of $L$ and $L_{1}$, the inclination of $K_{1}$ and $K_{2}$ is equal to that of $L_{1}$ and $L_{2}$, and so on, all these inclinations being otherwise arbitrary; or say that we have the double-triangle strips or elements $K K_{1}$, $K_{1} K_{2}, K_{2} K_{3}$, \&c., bounded by pairs of lines, at given infinitesimal inclinations the two lines of a pair to each other, and forming a flexible double pyramid, which may be bent into any given form whatever assumed at pleasure; and this being so, we see that the system of the strips or elements $L L_{1}, L_{1} L_{2}, L_{2} L_{3}$, \&c., may be so bent that the lines $L, L_{1}, L_{2}$, \&c., shall be parallel to the lines $K, K_{1}, K_{2}$, \&c., respectively.

Supposing the distances $P Q_{1}, P_{1} Q_{2}, P_{2} Q_{3}$, \&c., to be all of them infinitesimal, we have a skew surface containing upon it a curve $P_{1} P_{2} P_{3}$, \&c., which is the line of striction, viz. this is the locus of the point on a generating line which is the nearest point to the consecutive generating line. Tre line of striction cuts the several generating lines at an angle $\omega$, variable from line to line, which is called the obliquity; and the inclination between two consecutive generating lines is in a certain ratio to the shortest distance between the two lines. Let the inclination=shortest distance $\div \tau$, this magnitude $\tau$ being variable from line to line: its reciprocal $\tau^{-1}$ is called the "torsion"; so that the obliquity $\omega$ and the reciprocal of the torsion $\tau$ may be regarded as functions of $s$, the arc of the line of striction measured from any fixed point. The skew surface is thus composed of rigid strips or elements, each included between two consecutive lines. We have further seen that the surface may be bent by turning these rigid elements about the successive generating lines, in such wise that the generating lines become parallel to the generating lines of an arbitrary cone, which is called the "asymptotic" cone (otherwise the director cone); say the surface may be bent so that it shall have a given asymptotic cone.

I consider a given skew surface; I take $x, y, z$ for the coordinates of a point on the line of striction, and $\alpha, \beta, \gamma$ for the cosine-inclinations of the generating line through this point; $x, y, z, \alpha, \beta, \gamma$ are regarded as functions of $s$, the length of or distance along the line of striction measured from any fixed point thereof; and I use accents to denote differentiation in regard to $s$. We have

$$
\alpha^{2}+\beta^{2}+\gamma^{2}=1
$$


and therefore

also

and therefore

$$
\begin{aligned}
& \alpha \alpha^{\prime}+\beta \beta^{\prime}+\gamma \gamma^{\prime}=0 \\
& x^{\prime 2}+y^{\prime 2}+z^{\prime 2}=1
\end{aligned}
$$

$$
x^{\prime} x^{\prime \prime}+y^{\prime} y^{\prime \prime}+z^{\prime} z^{\prime \prime}=0
$$

$x^{\prime}, y^{\prime}, z^{\prime}$ are the cosine-inclinations of the tangent at the point $x, y, z$.

I remark also that, writing

$$
\rho^{-1}=\sqrt{x^{1 / 2}+y^{\prime \prime 2}+z^{1 / 2}}
$$

so that $\rho$ is the radius of absolute curvature, we have $\rho x^{\prime \prime}, \rho y^{\prime \prime}, \rho z^{\prime \prime}$ for the cosineinclinations of the binormal (or perpendicular to the osculating plane).

If from the point $(x, y, z)$ we draw a perpendicular to the consecutive generating line through the point $(x+d x, y+d y, z+d z)$, this will also be perpendicular to the generating line through the point $(x, y, z)$, and we thus find

$$
\alpha^{\prime} x^{\prime}+\beta^{\prime} y^{\prime}+\gamma^{\prime} z^{\prime}=0
$$

as the condition that the point $(x, y, z)$ may be, as it is assumed to be, a point on the line of striction.

For the proof hereof, take for the moment $x_{1}, y_{1}, z_{1}$ the coordinates of $P$, and $\alpha_{1}, \beta_{1}, \gamma_{1}$ for the cosine-inclinations of $L$; then, considering the line $P Q_{1}$, this passes through $P$ and cuts the line $L_{1}$; taking its equation to be

this meets the line

$$
\frac{X-x}{A}=\frac{Y-y}{B}=\frac{Z-z}{C},
$$

and we thence find

$$
\frac{X-x_{1}}{\alpha_{1}}=\frac{Y-y_{1}}{\beta_{1}}=\frac{Z-z_{1}}{\gamma_{1}}
$$

$$
\left|\begin{array}{ccc}
A, & B, & C \\
x_{1}-x, & y_{1}-y, & z_{1}-z \\
\alpha_{1}, & \beta_{1}, & \gamma_{1}
\end{array}\right|=0
$$

Writing $x_{1}, y_{1}, z_{1}=x+x^{\prime} d s, y+y^{\prime} d s, z+z^{\prime} d s$, $d s$ divides out, and we have

$$
A\left(\beta_{1} z^{\prime}-\gamma_{1} y^{\prime}\right)+B\left(\gamma_{1} x^{\prime}-\alpha_{1} z^{\prime}\right)+C\left(\alpha_{1} y^{\prime}-\beta_{1} x^{\prime}\right)=0 ;
$$

the line in question cuts $L_{1}$ and $L$; that is, we have

or, eliminating $A, B, C$, we find $A \alpha+B \beta+C \gamma=0$;

$$
\begin{aligned}
& A \alpha_{1}+B \beta_{1}+C \gamma_{1}=0 \\
& A \alpha+B \beta+C \gamma=0
\end{aligned}
$$

that is,

$$
\left(\beta \gamma_{1}-\beta_{1} \gamma\right)\left(\beta_{1} z^{\prime}-\gamma_{1} y^{\prime}\right)+\left(\gamma \alpha_{1}-\gamma_{1} \alpha\right)\left(\gamma_{1} x^{\prime}-\alpha_{1} z^{\prime}\right)+\left(\alpha \beta_{1}-\alpha_{1} \beta\right)\left(\alpha_{1} y^{\prime}-\beta_{1} x^{\prime}\right)=0
$$

$$
\left(\alpha_{1} x^{\prime}+\beta_{1} y^{\prime}+\gamma_{1} z^{\prime}\right)\left(\alpha \alpha_{1}+\beta \beta_{1}+\gamma \gamma_{1}\right)-\left(\alpha x^{\prime}+\beta y^{\prime}+\gamma z^{\prime}\right)\left(\alpha_{1}{ }^{2}+\beta_{1}{ }^{2}+\gamma_{1}^{2}\right)=0 .
$$

c. XIII. 
But we have

and, writing

$$
\alpha_{1}^{2}+\beta_{1}^{2}+\gamma_{1}^{2}=1
$$

we find

$$
\alpha_{1}, \beta_{1}, \gamma_{1}=\alpha+\alpha^{\prime} d s, \beta+\beta^{\prime} d s, \gamma+\gamma^{\prime} d s
$$

$$
\alpha \alpha_{1}+\beta \beta_{1}+\gamma \gamma_{1}=\alpha^{2}+\beta^{2}+\gamma^{2}+\left(\alpha \alpha^{\prime}+\beta \beta^{\prime}+\gamma \gamma^{\prime}\right) d s=1
$$

the equation thus is

that is,

$$
\left(\alpha_{1} x^{\prime}+\beta_{1} y^{\prime}+\gamma_{1} z^{\prime}\right)-\left(\alpha x^{\prime}+\beta y^{\prime}+\gamma z^{\prime}\right)=0
$$

the required equation.

$$
\alpha^{\prime} x^{\prime}+\beta^{\prime} y^{\prime}+\gamma^{\prime} z^{\prime}=0
$$

Calling the inclination of the generating line through the point $(x, y, z)$ to the tangent of the line of striction the "obliquity," and denoting it by $\omega$, we have

$$
\alpha x^{\prime}+\beta y^{\prime}+\gamma z^{\prime}=\cos \omega .
$$

Calling the inclination of the two consecutive generating lines divided by the shortest distance between these lines the "torsion," and denoting it by $\tau^{-1}$, we have

$$
\alpha^{\prime 2}+\beta^{\prime 2}+\gamma^{\prime 2}=\frac{\sin ^{2} \omega}{\tau^{2}}
$$

In proof hereof, if for a moment $\phi$ is the inclination of the lines $L$ and $L_{1}$ to each other, then

and therefore

$$
\cos \phi=\alpha \alpha_{1}+\beta \beta_{1}+\gamma \gamma_{1}
$$

viz. writing

$$
\sin ^{2} \phi=\left(\beta \gamma_{1}-\beta_{1} \gamma\right)^{2}+\left(\gamma \alpha_{1}-\gamma_{1} \alpha\right)^{2}+\left(\alpha \beta_{1}-\alpha_{1} \beta\right)^{2}
$$

this is

$$
\alpha_{1}, \beta_{1}, \gamma_{1}=\alpha+\alpha^{\prime} d s, \beta+\beta^{\prime} d s, \gamma+\gamma^{\prime} d s
$$

$$
\begin{aligned}
\sin ^{2} \phi & =d s^{2}\left\{\left(\beta \gamma^{\prime}-\beta^{\prime} \gamma\right)^{2}+\left(\gamma \alpha^{\prime}-\gamma^{\prime} \alpha\right)^{2}+\left(\alpha \beta^{\prime}-\alpha^{\prime} \beta\right)^{2}\right\} \\
& =d s^{2}\left\{\left(\alpha^{2}+\beta^{2}+\gamma^{2}\right)\left(\alpha^{\prime 2}+\beta^{\prime 2}+\gamma^{\prime 2}\right)-\left(\alpha \alpha^{\prime}+\beta \beta^{\prime}+\gamma \gamma^{\prime}\right)^{2}\right\} \\
& =d s^{2}\left(\alpha^{\prime 2}+\beta^{\prime 2}+\gamma^{\prime 2}\right)
\end{aligned}
$$

whence, if for a moment the shortest distance between the two lines is called $\delta$, then we have

$$
\frac{\sin \phi}{\delta}=\tau^{-1}=\frac{\sin \phi}{d s \sin \omega}
$$

that is,

$$
\frac{\sin ^{2} \omega}{\tau^{2}}=\frac{\sin ^{2} \phi}{d s^{2}}=\alpha^{\prime 2}+\beta^{\prime 2}+\gamma^{\prime 2}
$$

the required equation. 
We have thus the five equations

$$
\begin{aligned}
\alpha^{2}+\beta^{2}+\gamma^{2} & =1, \\
x^{\prime 2}+y^{\prime 2}+z^{\prime 2} & =1, \\
\alpha^{\prime} x^{\prime}+\beta^{\prime} y^{\prime}+\gamma^{\prime} z^{\prime} & =0 \\
\alpha x^{\prime}+\beta y^{\prime}+\gamma z^{\prime} & =\cos \omega \\
\alpha^{\prime 2}+\beta^{\prime 2}+\gamma^{\prime 2} & =\frac{\sin ^{2} \omega}{\tau^{2}}
\end{aligned}
$$

and if we herein consider $\omega$ and $\tau$ as denoting given functions of $s$, all the skew surfaces which satisfy these equations will be surfaces applicable one on the other.

Adding to the foregoing the derived equations

$$
\begin{gathered}
\alpha \alpha^{\prime}+\beta \beta^{\prime}+\gamma \gamma^{\prime}=0, \\
x^{\prime} x^{\prime \prime}+y^{\prime} y^{\prime \prime}+z^{\prime} z^{\prime \prime}=0, \\
\alpha x^{\prime \prime}+\beta y^{\prime \prime}+\gamma z^{\prime \prime}=-\sin \omega \cdot \omega^{\prime}, \\
\alpha x^{\prime \prime \prime}+\beta y^{\prime \prime \prime}+\gamma z^{\prime \prime \prime}+\alpha^{\prime} x^{\prime \prime}+\beta^{\prime} y^{\prime \prime}+\gamma^{\prime} z^{\prime \prime}=-\sin \omega \cdot \omega^{\prime \prime}-\cos \omega \cdot \omega^{\prime 2},
\end{gathered}
$$

we find without difficulty

$$
\begin{gathered}
\alpha^{\prime}, \beta^{\prime}, \gamma^{\prime}=\frac{\beta z^{\prime}-\gamma y^{\prime}}{\tau}, \frac{\gamma x^{\prime}-\alpha z^{\prime}}{\tau}, \frac{\alpha y^{\prime}-\beta x^{\prime}}{\tau}, \\
\beta \gamma^{\prime}-\beta^{\prime} \gamma, \gamma \alpha^{\prime}-\gamma^{\prime} \alpha, \alpha \beta^{\prime}-\alpha^{\prime} \beta=\frac{-x^{\prime}+\alpha \cos \omega}{\tau}, \frac{-y^{\prime}+\beta \cos \omega}{\tau}, \frac{-z^{\prime}+\gamma \cos \omega}{\tau} .
\end{gathered}
$$

Putting, for shortness,

and, as above,

$$
\begin{gathered}
\Theta=-\sin \omega \cdot \omega^{\prime \prime}-\cos \omega \cdot \omega^{2}-\left(\alpha x^{\prime \prime \prime}+\beta y^{\prime \prime \prime}+\gamma z^{\prime \prime}\right) \\
\nabla=\left|\begin{array}{ccc}
\alpha, & \beta, & \gamma \\
x^{\prime}, & y^{\prime}, & z^{\prime} \\
x^{\prime \prime}, & y^{\prime \prime}, & z^{\prime \prime}
\end{array}\right|
\end{gathered}
$$

we find

$$
\rho^{-2}=x^{\prime \prime 2}+y^{\prime \prime 2}+z^{\prime \prime 2}
$$

$$
\nabla^{2}=\sin ^{2} \omega\left(\frac{1}{\rho^{2}}-\omega^{\prime 2}\right), \quad \tau=\frac{\nabla}{\Theta}=\frac{\sin \omega}{\rho \Theta} \sqrt{1-\rho^{2} \omega^{\prime 2}},
$$

and to these may be joined

$$
\begin{aligned}
& \beta z^{\prime}-\gamma y^{\prime}=\rho^{2}\left\{\nabla x^{\prime \prime}+\omega^{\prime} \sin \omega\left(y^{\prime} z^{\prime \prime}-y^{\prime \prime} z^{\prime}\right)\right\} \\
& \gamma x^{\prime}-\alpha z^{\prime}=\rho^{2}\left\{\nabla y^{\prime \prime}+\omega^{\prime} \sin \omega\left(z^{\prime} x^{\prime \prime}-z^{\prime \prime} x^{\prime}\right)\right\} \\
& \alpha y^{\prime}-\beta x^{\prime}=\rho^{2}\left\{\nabla z^{\prime \prime}+\omega^{\prime} \sin \omega\left(x^{\prime} y^{\prime \prime}-x^{\prime \prime} y^{\prime}\right)\right\}
\end{aligned}
$$


I remark that, supposing the line of striction to be given, that is, $(x, y, z)$ to be given as functions of $s$, and moreover the obliquity $\omega$ to be given as a function of $s$, the position of the generating line through the point $(x, y, z)$ will be given, and also the torsion $\tau^{-1}$. In fact, among the foregoing equations, we have

$$
\begin{aligned}
& \alpha^{2}+\beta^{2}+\gamma^{2}=1, \\
& \alpha x^{\prime}+\beta y^{\prime}+\gamma z^{\prime}=\cos \omega, \\
& \alpha x^{\prime \prime}+\beta y^{\prime \prime}+\gamma z^{\prime \prime}=-\sin \omega \cdot \omega^{\prime},
\end{aligned}
$$

which equations determine $\alpha, \beta, \gamma$, that is, the position of the generating line; the position of the consecutive generating line is then also determined; we thus have $\alpha^{\prime}, \beta^{\prime}, \gamma^{\prime}$, and thence the torsion, which is given by the foregoing equation

$$
\tau=\frac{\sin \omega}{\rho \Theta} \sqrt{1-\rho^{2} \omega^{\prime 2}} .
$$

Supposing that the line of striction is not given, but that the obliquity $\omega$ and the torsion $\tau^{-1}$ are given as functions of $s$, we have then the foregoing five equations, which are not sufficient for the determination of $x, y, z, \alpha, \beta, \gamma$; but, joining to them an assumed homogeneous relation between $(\alpha, \beta, \gamma)$, the six quantities will be determined. The assumed homogeneous relation between $(\alpha, \beta, \gamma)$ is the equation of the asymptotic cone; and we have thus the theorem that, given the asymptotic cone, and also the obliquity and the torsion as functions of $s$ (the are of the line of striction), then this line of striction, and the skew surface the locus of the generating lines, will be determined.

We have between $\alpha, \beta, \gamma$ the assumed homogeneous equation, say $U=0$; and among the foregoing equations, the equations

$$
\alpha^{2}+\beta^{2}+\gamma^{2}=1, \text { and } \alpha^{\prime 2}+\beta^{\prime 2}+\gamma^{\prime 2}=\frac{\sin ^{2} \omega}{\tau^{2}},
$$

a given function of $s$; these equations give, by means of an integration, $\alpha, \beta, \gamma$ as functions of $s$; and we have then the above-mentioned equations

$$
x^{\prime}-\alpha \cos \omega, y^{\prime}-\beta \cos \omega, z^{\prime}-\gamma \cos \omega=\tau\left(\beta \gamma^{\prime}-\beta^{\prime} \gamma, \gamma \alpha^{\prime}-\gamma^{\prime} \alpha, \alpha \beta^{\prime}-\alpha^{\prime} \beta\right),
$$

which give, by integration, $x, y, z$ as functions of $s$. And the skew surfaces thus obtained for an assumed asymptotic cone $U=0$ will be the required system of skew surfaces applicable the one on the other.

For example, if $\omega, \tau$ are constants, and we take, for $U=0$, the equation

$$
\gamma^{2}-c\left(\alpha^{2}+\beta^{2}\right)=0
$$

that is, if we consider the skew surfaces of constant obliquity and torsion and which have for asymptotic cone a right circular cone: it easily appears that the line of striction is a helix traced upon a right circular cylinder, and that the generating lines are at a constant inclination to the axis of the cylinder, and all of them touch the cylinder. In fact, for such a surface (a kind of helicoid), it is in the first 
place obvious that the helix is the line of striction, and next that the obliquity and the torsion are each of them constant.

Supposing that the obliquity and the torsion are $\omega, \tau$, and that the inclination of the generating lines to the axis of the cylinder is $90^{\circ}-\omega-\delta, \delta$ being a given constant, we find

$$
\begin{aligned}
& x=\frac{\tau \cos \delta \cos (\omega+\delta)}{\sin \omega} \cos \left\{\frac{s \sin \omega}{\tau \cos (\omega+\delta)}\right\} \\
& y=\frac{\tau \cos \delta \cos (\omega+\delta)}{\sin \omega} \sin \left\{\frac{s \sin \omega}{\tau \cos (\omega+\delta)}\right\} \\
& z=s \sin \delta \\
& \alpha=-\cos (\omega+\delta) \sin \left\{\frac{s \sin \omega}{\tau \cos (\omega+\delta)}\right\} \\
& \beta=\cos (\omega+\delta) \cos \left\{\frac{s \sin \omega}{\tau \cos (\omega+\delta)}\right\} \\
& \gamma=\sin (\omega+\delta)
\end{aligned}
$$

where observe that the line of striction lies on the circular cylinder,

$$
\text { radius }=\tau \cos \delta \cos (\omega+\delta) \div \sin \omega \text {. }
$$

In particular, if $\delta=0$, we have

$$
\begin{array}{ll}
x=\tau \cot \omega \cos \frac{s}{\tau \cot \omega}, & \alpha=-\cos \omega \sin \frac{s}{\tau \cot \omega}, \\
y=\tau \cot \omega \sin \frac{s}{\tau \cot \omega}, & \beta=\cos \omega \cos \frac{s}{\tau \cot \omega}, \\
z=0, & \gamma=\sin \omega ;
\end{array}
$$

and, for $\delta=-\omega$, we have

$$
\begin{array}{ll}
x=\tau \cot \omega \cos \frac{s \sin \omega}{\tau}, & \alpha=-\sin \frac{s \sin \omega}{\tau}, \\
y=\tau \cot \omega \sin \frac{s \sin \omega}{\tau}, & \beta=\cos \frac{s \sin \omega}{\tau}, \\
z=-s \sin \omega, & \gamma=0 .
\end{array}
$$

The first of these is the skew hyperboloid of revolution

$$
X^{2}+Y^{2}-\left(Z^{2}+\tau^{2}\right) \cot ^{2} \omega=0,
$$

(radius of gorge $=\tau \cot \omega$ ); and the second of them is the helicoid (radius of cylinder $=\tau \cot \omega)$, the generating lines of which are the tangents perpendicular to the axis of the cylinder; these are, in fact, surfaces found by Bonnet in his memoir above referred to. We may imagine the surface passing from the first form, in which the semi-aperture of the asymptotic cone is $\omega$, through the series of forms belonging to the general formulæ involving $\delta$, to the second form, in which the semi-aperture is $=90^{\circ}$, i.e. in which the asymptotic cone is replaced by a plane. 